\title{
Norbornene probes for the detection of cysteine sulfenic acid in cells
}

\author{
Lisa J. Alcock,${ }^{\mathrm{a}, \mathrm{b}}$ Bruno L. Oliveira, ${ }^{\mathrm{c}}$ Michael J. Deery, ${ }^{\mathrm{d}}$ Tara L. Pukala, ${ }^{\mathrm{e}}$ Michael V. Perkins, ${ }^{\mathrm{a}}$ \\ Gonçalo J. L. Bernardes, ${ }^{\text {c,f* }}$ and Justin M. Chalker ${ }^{\mathrm{a}, \mathrm{b}^{*}}$ \\ ${ }^{a}$ Flinders University, College of Science and Engineering, Sturt Road, Bedford Park, South Australia 5042, Australia \\ ${ }^{\mathrm{b}}$ Flinders University, Institute for NanoScale Science and Technology, Sturt Road, Bedford Park, South Australia 5042 , \\ Australia \\ ${ }^{\mathrm{c}}$ University of Cambridge, Department of Chemistry, Lensfield Road, Cambridge CB2 1EW, UK \\ ${ }^{\mathrm{d}}$ Cambridge Centre for Proteomics, Cambridge Systems Biology Centre, Department of Biochemistry, University of \\ Cambridge, Tennis Court Road, Cambridge CB2 1QW, UK \\ ${ }^{\mathrm{e}}$ The University of Adelaide, School of Physical Sciences, Adelaide, South Australia 5005, Australia \\ ${ }_{\mathrm{f}}^{\mathrm{f}}$ Instituto de Medicina Molecular, Faculdade de Medicina, Universidade de Lisboa, Avenida Professor Egas Moniz, \\ 1649-028, Lisboa, Portugal
}

\begin{abstract}
Norbornene derivatives were validated as probes for cysteine sulfenic acid on proteins and in live cells. Trapping sulfenic acids with norbornene probes is highly selective and revealed a different reactivity profile than the traditional dimedone reagent. The norbornene probe also revealed a superior chemoselectivity when compared to a commonly used dimedone probe. Together, these results advance the study of cysteine oxidation in biological systems.
\end{abstract}

Cysteine residues on proteins react with cellular oxidants such as hydrogen peroxide, superoxide, and peroxynitrite. $^{1,2}$ One product of these reactions, cysteine sulfenic acid (Fig. 1a), is linked to a variety of redox regulation mechanisms and signalling pathways, and may serve as a biomarker for oxidative stress. ${ }^{3}$ To understand the cellular and physiological roles of cysteine sulfenic acid, it is important to be able to detect its formation on proteins. ${ }^{1,2}$ Such analyses are challenging because cysteine sulfenic acids are sometimes short-lived. Additionally, selective detection of the sulfenic acid oxidation state must occur in the presence of thiols, disulfides, sulfinic acids and other derivatives of cysteine.

The standard reagent used for detecting cysteine sulfenic acids is dimedone (5,5-dimethyl-1,3-cyclohexanedione) ${ }^{4}$ and derivatives labeled with biotin, ${ }^{5,}{ }^{6}$ fluorophores, ${ }^{6}$ alkynes, ${ }^{7}$ or azides. ${ }^{8,9}$ While dimedone probes are reported to be selective for the sulfenic acid oxidation state of cysteine, they often react slowly. ${ }^{10,} 11$ To increase the reactivity of sulfenic acid probes, a number of structural variations of dimedone and carbon-centered nucleophiles have been explored, ${ }^{10,12,13}$ but there remains a need for probes that react both rapidly and selectively with cysteine sulfenic acid.

Recently, we investigated norbornene derivatives as cysteine sulfenic acid traps. ${ }^{11}$ In this strategy, the olefin reacts with the sulfenic acid through a strain-promoted group transfer reaction (Fig. 1a). This reaction was validated on small-molecule models, which revealed a higher reaction rate than dimedone. ${ }^{11}$ A similar strategy has also been explored using cyclooctynes to trap sulfenic acids, ${ }^{14,15}$ but these highly strained probes suffer from offtarget reactions, particularly with biological thiols. ${ }^{11,16,17}$ With less strain, norbornene is more selective than cyclooctynes. ${ }^{11}$ Another benefit of the norbornene scaffold is that it is straightforward to synthesise and modify, enabling access to biotin- and alkyne-tagged derivatives such as $\mathbf{1}$ and $\mathbf{2}$ (norb-bio and norb-yne, respectively, Fig. 1b). ${ }^{11}$

Motivated by these promising small-molecule studies, ${ }^{11}$ we carried out the first evaluation of norbornene derivatives as probes for cysteine sulfenic acid on proteins. Cysteine sulfenic acid was detected on a model protein, in HeLa cell lysates, and in living HeLa cells. Probes $\mathbf{1}$ and $\mathbf{2}$ displayed a different reactivity profile and superior selectivity when compared to a widely used dimedone probe-providing a new tool for the selective detection and analysis of cysteine sulfenic acid.

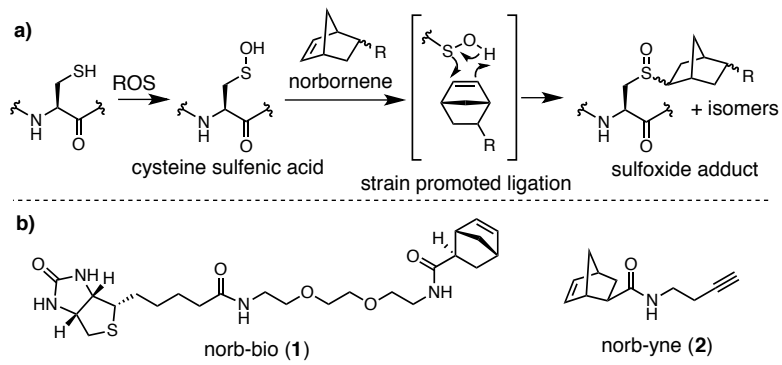

Figure 1. a) Strain-promoted ligation of norbornene with sulfenic acids. (ROS $=$ reactive oxygen species) b) Norbornene probes containing detectable tags. ${ }^{11}$

Norbornene derivative 3 was first tested on the cysteine protease papain. Papain has a single free cysteine in its active site (Cys25) that can be oxidized with hydrogen peroxide ${ }^{18}$ to generate a model cysteine sulfenic acid. ${ }^{4,6,8}$ After optimization studies (S2-S12), papain was treated with probe 3 before adding $\mathrm{H}_{2} \mathrm{O}_{2}$ (Fig. 2a). After incubating for 1 hour at room temperature, the excess probe and oxidant were removed using a centrifugal concentrator. 
The protein was then analyzed by liquid chromatographymass spectrometry (LC-MS, Fig. 2b). Two clear signals were observed in the deconvoluted mass spectrum (Fig. $2 \mathrm{~b}$ ). The signal at $23619 \mathrm{Da}$ is consistent with sulfoxide adduct 4, expected to form after reaction of the probe with the sulfenic acid of papain. This was the first demonstration of norbornene derivatives as cysteine sulfenic acid traps on proteins. The signal at $23457 \mathrm{Da}$ is consistent with conversion of the active site cysteine to the sulfinic acid (5), a product of over-oxidation (S5). Importantly, no reaction of $\mathbf{3}$ was observed with papain in the absence of $\mathrm{H}_{2} \mathrm{O}_{2}$ (S6), demonstrating selective reaction of the norbornene probe with the sulfenic acid oxidation state of cysteine.

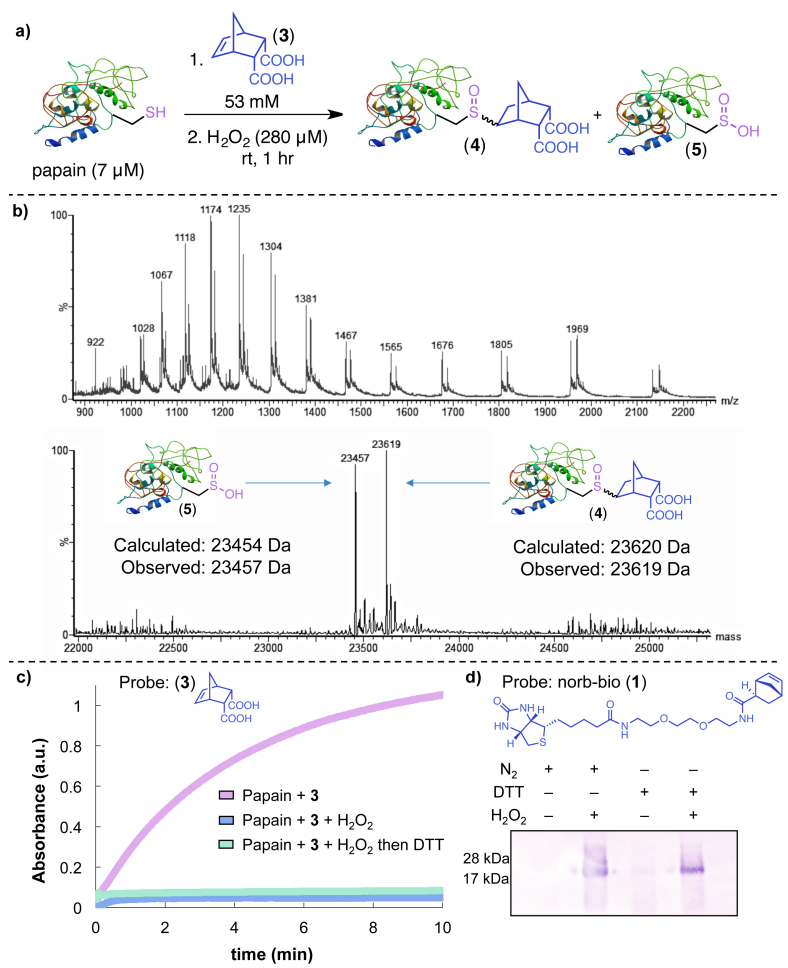

Figure 2. a) Reaction of papain with $\mathrm{H}_{2} \mathrm{O}_{2}$, in the presence of probe 3. b) LC-MS analysis revealing the detection of sulfoxide 4 and sulfinic acid 5. c) Activity assay of papain. Probe 3 reacts with and inhibits papain only in the presence of $\mathrm{H}_{2} \mathrm{O}_{2}$. d) Western blot of papain after treatment with $\mathrm{H}_{2} \mathrm{O}_{2}(2 \mathrm{mM})$ in the presence of biotin-labeled probe $\mathbf{1}(10 \mathrm{mM})$.

Reaction at the active site cysteine of papain was consistent with enzyme activity assays (Fig. 2c and S10S12). Treating papain $(7 \mu \mathrm{M})$ with $\mathrm{H}_{2} \mathrm{O}_{2}(2.8 \mathrm{mM})$ lead to oxidation and complete loss of enzyme activity (S10S12). ${ }^{19}$ Treating the oxidized papain with reducing agent dithiothreitol (DTT), lead to partial recovery of activity (S10-S12). This result is consistent with the formation of cysteine sulfenic acid and higher oxidation states of cysteine and then reduction of the cysteine sulfenic acid back to the active thiol. In contrast, when norbornene derivative 3 was present during the oxidation at a concentration of $53 \mathrm{mM}$, no enzyme activity could be recovered using DTT because of the formation of the stable sulfoxide adduct 4 (Fig. 2c). Importantly, the activity of papain was not affected by $\mathbf{3}$ in the absence of $\mathrm{H}_{2} \mathrm{O}_{2}$ (Fig. 2c), indicating it did not react with the active site thiol.

Biotin-labeled norbornene derivative 1 was also evaluated on papain. In the event, papain $(15 \mu \mathrm{M})$ was incubated with $1(10 \mathrm{mM})$ both with and without $\mathrm{H}_{2} \mathrm{O}_{2}$ (2 $\mathrm{mM}$ ) for $1 \mathrm{~h}$ at room temperature. These reactions were carried out under an atmosphere of $\mathrm{N}_{2}$ or in the presence of DTT to prevent air oxidation of Cys25 (S4, S12-15). ${ }^{20}$ These reactions were analyzed by SDS-PAGE and western blotting. Probe 1 only labeled papain in the presence of $\mathrm{H}_{2} \mathrm{O}_{2}$ (Fig. 2d).

With the norbornene probes validated on a protein model, cell lysates were examined next. To determine optimal labeling conditions, the concentration of $\mathrm{H}_{2} \mathrm{O}_{2}$ and probes 1 and $\mathbf{2}$ were systematically varied (S15-S20). Accordingly, $\mathrm{HeLa}$ cell lysates were treated with the norbornene probe $\left(0.1\right.$ to $3 \mathrm{mM}$ ), followed by $\mathrm{H}_{2} \mathrm{O}_{2}$ ( 0 to 2 $\mathrm{mM})$ for $1 \mathrm{~h}$ at room temperature. Samples were then purified with size exclusion columns to remove excess probe. For probe 1, samples were analyzed directly by SDS-PAGE and western blotting. For probe 2, samples were ligated to an azide-tagged biotin derivative prior to SDS-PAGE (Fig. 3a-b). Labeling profiles were also compared to the widely used dimedone derivative DCPBiol (6) using the same protocol (Fig. 3c). ${ }^{6,21}$

For both norbornene probes, increased labeling was observed with increased probe concentration and increased $\mathrm{H}_{2} \mathrm{O}_{2}$ concentration (Fig. 3a-b). As a control, lysates were also pre-treated with $\mathrm{H}_{2} \mathrm{O}_{2}(2 \mathrm{mM})$ for 30 minutes before addition of the norbornene probes. In this case, no significant protein labeling was observed. Overoxidation to the sulfinic or sulfonic acids is likely, neither of which react with the norbornene-based probes ${ }^{11}$ (Fig. 3a-b, far right lane). When the norbornene probes were added to lysate pre-treated with the reducing agent tris(2carboxyethyl)phosphine (TCEP), no labeling was observed because any endogenous sulfenic acid would be reduced by TCEP (Fig 3a-b, far left lane). This outcome also indicates that the norbornene probe does not react with cellular thiols under the conditions of the assay. Together, the results in Figure 3 indicate that norbornene probes are highly selective for cysteine sulfenic acid, even in a complex protein mixture. Non-selective labeling was only observed if excess 1 was not removed before denaturing proteins at elevated temperatures $\left(\sim 95{ }^{\circ} \mathrm{C}, \mathrm{S} 22\right)$. Under these conditions it is possible that the norbornene probes can react directly with thiols through a thiol-ene reaction. Therefore, to avoid these false positives, it is important to remove excess probe with a desalting column or sizeexclusion chromatography before denaturing proteins for SDS-PAGE analysis.

Interestingly, dimedone probe $\mathbf{6}$ led to a different labeling profile than norbornene probes 1 and 2 (Fig. 3c). The different protein hits may reflect the different size and distinct reaction mechanisms of the norbornene and dimedone probes. Dimedone probe $\mathbf{6}$ also labeled proteins in the control experiment in which the lysates were pretreated with $\mathrm{H}_{2} \mathrm{O}_{2} 30$ minutes before the addition of the probe (Fig. 3c, far right lane). It was thought that under these conditions cysteine sulfenic acids would be overoxidized before the addition of the probe, so $\mathbf{6}$ may react unselectively with other residues in this situation. It is known, for instance, that dimedone can react with amines, ${ }^{22}$ even at room temperature under certain conditions, ${ }^{23}$ but it is not clear what is causing the nonselective labeling in this experiment. Non-selective labeling was also observed when 6 was added to cell lysates pre-treated with the reducing agent TCEP (Fig. 3c, far left lane). Previous studies have indicated that $\mathbf{6}$ can label proteins non-selectively if cysteine thiols are not alkylated. $^{21}$ The presence of TCEP apparently exacerbates this problem by increasing thiol concentration through 
reduction of protein disulfides. A similar outcome was observed if DTT was used in the pre-reduction-again leading to non-selective labelling by dimedone derivative 6 (S23-S24). Even with attempts to remove excess 6 before SDS-PAGE analysis, off-target labelling was observed (S23-S24). These results indicate dimedone probe $\mathbf{6}$ is not as selective as norbornene derivatives $\mathbf{1}$ and 2. These results also prompt a cautionary note that false positives are possible when using probe $\mathbf{6}$ in the presence of free thiols or in experiments in which reducing conditions are established by adding TCEP or DTT to protein mixtures.

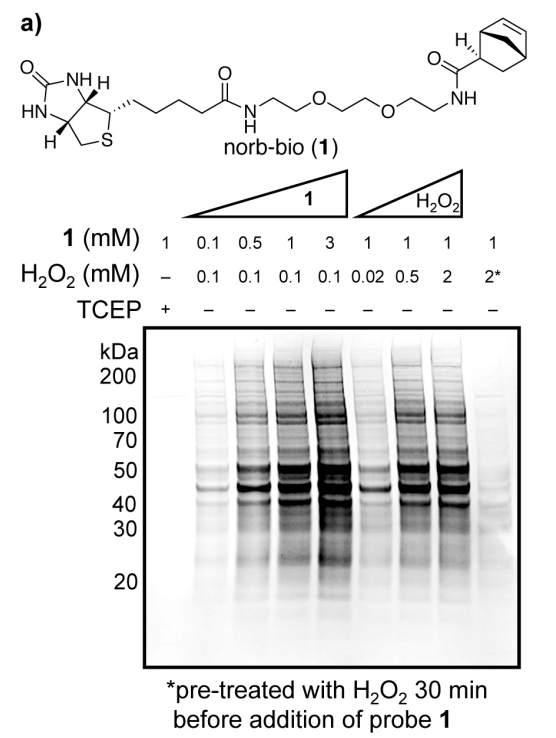

b)

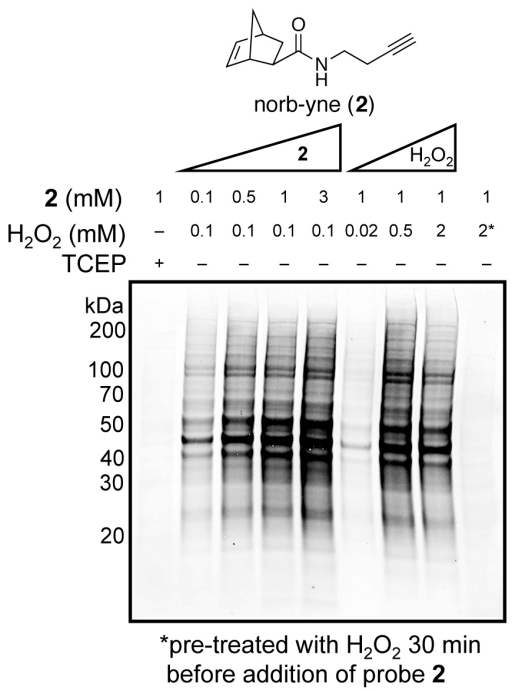

c)

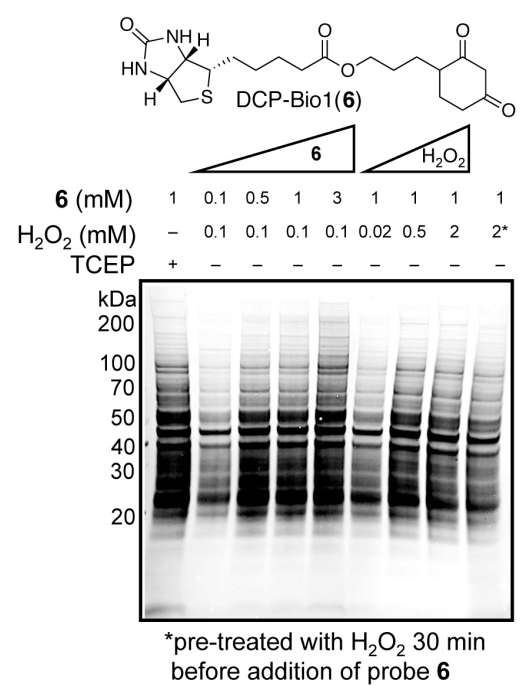

Figure 3. Assessment of 1, 2, and $\mathbf{6}$ as probes for cysteine sulfenic acid in HeLa cell lysates. Probes were added to the lysates as solutions in DMSO before the addition of $\mathrm{H}_{2} \mathrm{O}_{2}$. The reactions were carried out for 1 hour at room temperature. All samples were purified using a size exclusion spin column before SDS-PAGE analysis and western blotting. Probe 2 was modified with a biotin-containing azide before SDS-PAGE. Western blots were developed using an Alexa Fluor 555 streptavidin conjugate.

Returning to the evaluation of the norbornene probes, proteomics analysis was used to confirm the site of labeling for representative hits. Accordingly, after labeling cell lysates with probe 2 , the $45 \mathrm{kDa}$ band was excised, digested with chymotrypsin, and analyzed by LC-MS/MS (S25). This band was selected because it was a prominent signal for both probes $\mathbf{1}$ and $\mathbf{2}$ in the western blot. The expected product formed from the reaction of $\mathbf{2}$ with cysteine sulfenic acid was indeed detected on two separate actin proteins (S25), providing additional confirmation of the site- and chemoselectivity of the norbornene probe.

The norbornene probes were tested in living cells next. The toxicity of probes $\mathbf{1}$ and 2, the DMSO vehicle, and $\mathrm{H}_{2} \mathrm{O}_{2}$ were first assessed over a 5 hour exposure period to help identify conditions suitable for live cell analysis. The concentration of 1 was tolerated up to $3 \mathrm{mM}$, with $75-80 \%$ viability over 72 hours after replacing the growth media (S26). Probe 2, however, had limited solubility at $3 \mathrm{mM}$ and was toxic to cells, leading to complete loss of cell viability (S26). Therefore, probe $\mathbf{2}$ was not considered further. The DMSO vehicle was tolerated up to $1 \%$ by volume, with $80 \%$ cell viability (S26). Generally, DMSO concentrations higher than $0.5 \%$ by volume can be toxic to cells. ${ }^{24}$ However, many live cell sulfenic acid studies have used DMSO concentrations up to $2 \%{ }^{7},{ }^{9} \mathrm{H}_{2} \mathrm{O}_{2}$ was tolerated by cells up to $0.33 \mathrm{mM}$, but $1 \mathrm{mM} \mathrm{H}_{2} \mathrm{O}_{2}$ led to total cell death after 5 hours of exposure to the oxidant (S26). Similar toxicity levels of $\mathrm{H}_{2} \mathrm{O}_{2}$ have been reported previously, but the values vary widely. ${ }^{25}$ In these cases, cells are most likely dying due to extreme oxidative stress. $^{26}$

Guided by this toxicity data, the labeling of cysteine sulfenic acid residues was tested in live cells (Fig. 4 and S27-S29). In these experiments, HeLa cells were treated with a solution of $\mathbf{1}$ in DMSO so that the probe concentration was 1 or $3 \mathrm{mM}$ and the final concentration of DMSO was $0.33 \%$ or $1 \%$ by volume, respectively. The cells were then incubated for 2 hours at $37{ }^{\circ} \mathrm{C}$ before the addition of $\mathrm{H}_{2} \mathrm{O}_{2}$. This initial incubation period was designed to allow the probe to enter the cell before inducing oxidative stress. After the addition of the $\mathrm{H}_{2} \mathrm{O}_{2}$, the cells were incubated for an additional 2 hours before they were harvested, washed, and lysed. The protein lysate was then analyzed by SDS-PAGE and western blotting. Protein labeling increased with increasing concentration of 1 and labeling was only observed in the presence of hydrogen peroxide (Fig. 4). With increasing concentrations of $\mathrm{H}_{2} \mathrm{O}_{2}(0.5-2.0 \mathrm{mM})$, labeling also increased (S27-S28). In contrast to the cell lysate experiments in Figure 3, the same labeling was observed whether or not the protein mixtures were passed through a size exclusion column before SDS-PAGE and western blotting, so this precaution does not seem to be necessary for live cell experiments (S29). We are currently embarked on a dedicated proteomics study to annotate these hits and evaluate them against the known sulfenome.

In summary, norbornene probes were evaluated in the detection of cysteine sulfenic acid on proteins and in cells. The norbornene probes are straightforward to prepare and selectively react with the sulfenic acid oxidation state of cysteine. The norbornene probes were more selective than commonly used dimedone probe $\mathbf{6}$. These studies will help advance the understanding of cysteine oxidation and its role in oxidative stress and redox signaling. 


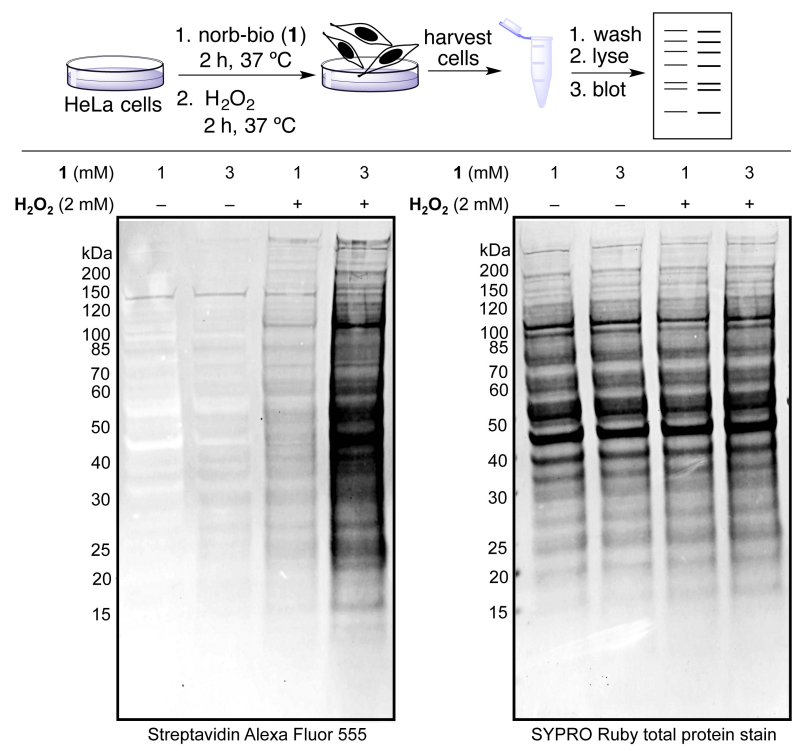

Figure 4. Norb-bio (1) was used to detect cysteine sulfenic acid residues in live HeLa cells. Significant labeling was only observed in the presence of $\mathrm{H}_{2} \mathrm{O}_{2}$, using $3 \mathrm{mM}$ probe. Total protein staining is shown on the right, indicating the protein concentration was controlled in these experiments.

\section{ASSOCIATED CONTENT}

\section{Supporting Information.}

The Supporting Information is available free of charge on the ACS Publications website. This file includes full experimental details and characterization data (pages S1-S29).

\section{Corresponding Authors}

*justin.chalker@flinders.edu.au *gb453@cam.ac.uk

\section{ACKNOWLEDGMENT}

The authors acknowledge generous financial support from the Australian Research Council (DE150101863, J.M.C.), The Royal Society (UF110046 and URF $\backslash R \backslash 180019$ ， G.J.L.B.), FCT Portugal (iFCT, IF/00624/2015, G.J.L.B.), the European Research Council (Starting Grant No. 676832, G.J.L.B.), Marie Sklodowska-Curie ITN Protein Conjugates (No. 67500, G.J.L.B), and the EPSRC (G.J.L.B.). L.J.A. is supported by an Australian Government Endeavour Postgraduate Scholarship.

\section{REFERENCES}

(1) Alcock, L. J., Perkins, M. V., and Chalker, J. M. (2018) Chemical methods for mapping cysteine oxidation, Chem. Soc. Rev. 47, 231-268.

(2) Paulsen, C. E., and Carroll, K. S. (2013) Cysteine-Mediated Redox Signaling: Chemistry, Biology, and Tools for Discovery, Chem. Rev. 113, 4633-4679.

(3) Poole, L. B., Karplus, P. A., and Claiborne, A. (2004) Protein Sulfenic Acids in Redox Signaling, Annu. Rev. Pharmacol. Toxicol. 44, 325-347.

(4) Benitez, L. V., and Allison, W. S. (1974) The Inactivation of the Acyl Phosphatase Activity Catalyzed by the Sulfenic Acid Form of Glyceraldehyde 3-Phosphate Dehydrogenase by Dimedone and Olefins, J. Biol. Chem. 249, 6234-6243.

(5) Charles, R. L., Schroder, E., May, G., Free, P., Gaffney, P. R. J., Wait, R., Begum, S., Heads, R. J., and Eaton, P. (2007) Protein sulfenation as a redox sensor-proteomics studies using a novel biotinylated dimedone analogue, Mol. Cell. Proteomics 6, 1473-1484.
(6) Poole, L. B., Klomsiri, C., Knaggs, S. A., Furdui, C. M., Nelson, K. J., Thomas, M. J., Fetrow, J. S., Daniel, L. W., and King, S. B. (2007) Fluorescent and affinity-based tools to detect cysteine sulfenic acid formation in proteins, Bioconjugate Chem. 18, 2004-2017.

(7) Paulsen, C. E., Truong, T. H., Garcia, F. J., Homann, A., Gupta, V., Leonard, S. E., and Carroll, K. S. (2012) Peroxidedependent sulfenylation of the EGFR catalytic site enhances kinase activity, Nat. Chem. Biol. 8, 57-64.

(8) Reddie, K. G., Seo, Y. H., Muse III, W. B., Leonard, S. E., and Carroll, K. S. (2008) A chemical approach for detecting sulfenic acid-modified proteins in living cells, Mol. BioSyst. 4, 521-531.

(9) Leonard, S. E., Reddie, K. G., and Carroll, K. S. (2009) Mining the thiol proteome for sulfenic acid modifications reveals new targets for oxidation in cells, ACS Chem. Biol. 4, 783-799.

(10) Gupta, V., and Carroll, K. S. (2016) Profiling the reactivity of cyclic C-nucleophiles towards electrophilic sulfur in cysteine sulfenic acid, Chem. Sci. 7, 400-415.

(11) Alcock, L. J., Farrell, K. D., Akol, M. T., Jones, G. H., Tierney, M. M., Kramer, H. B., Pukala, T. L., Bernardes, G. J. L., Perkins, M. V., and Chalker, J. M. (2018) Norbornene probes for the study of cysteine oxidation, Tetrahedron 74, 1220-1228.

(12) Gupta, V., Yang, J., Liebler, D. C., and Carroll, K. S. (2017) Diverse Redoxome Reactivity Profiles of Carbon Nucleophiles, J. Am. Chem. Soc. 139, 5588-5595.

(13) Gupta, V., and Carroll, K. S. (2016) Rational design of reversible and irreversible cysteine sulfenic acid-targeted linear C-nucleophiles, Chem. Commun. 52, 3414-3417.

(14) Poole, T. H., Reisz, J. A., Zhao, W., Poole, L. B., Furdui, C. M., and King, S. B. (2014) Strained Cycloalkynes as New Protein Sulfenic Acid Traps, J. Am. Chem. Soc. 136, 6167-6170.

(15) McGarry, D. J., Shchepinova, M. M., Lilla, S., Hartley, R. C., and Olson, M. F. (2016) A Cell-Permeable Biscyclooctyne As a Novel Probe for the Identification of Protein Sulfenic Acids, ACS Chem. Biol. 11, 3300-3304.

(16) Galardon, E., and Padovani, D. (2015) Reactivity of Persulfides Toward Strained Bicyclo[6.1.0]nonyne Derivatives: Relevance to Chemical Tagging of Proteins, Bioconjugate Chem. 26, 1013-1016.

(17) van Geel, R., Pruijn, G. J. M., van Delft, F. L., and Boelens, W. C. (2012) Preventing Thiol-Yne Addition Improves the Specificity of Strain-Promoted Azide-Alkyne Cycloaddition, Bioconjugate Chem. 23, 392-398.

(18) Lin, W. S., Armstrong, D. A., and Gaucher, G. M. (1975) Formation and Repair of Papain Sulfenic Acid, Can. J. Biochem. 53, 298-307.

(19) Sanner, T., Pihl, A. (1962) Studies on the Active -SH Group of Papain and on the Mechanism of Papain Activation by Thiol, J. Biol. Chem. 238, 165-171.

(20) Sluyterman, L. A. Æ. (1967) The activation reaction of papain, Biochim. Biophys. Acta, Enzymol. 139, 430-438.

(21) Klomsiri, C., Nelson, K. J., Bechtold, E., Soito, L., Johnson, L. C., Lowther, W. T., Ryu, S.-E., King, S. B., Furdui, C. M., and Poole, L. B. (2010) Use of Dimedone-Based Chemical Probes for Sulfenic Acid Detection: Evaluation of Conditions Affecting Probe Incorporation into Redox-Sensitive Proteins, Methods Enzymol. 473, 77-94.

(22) Poole, L. B., Zeng, B.-B., Knaggs, S. A., Yakubu, M., and King, S. B. (2005) Synthesis of chemical probes to map sulfenic acid modifications on proteins, Bioconjugate Chem. 16, 16241628.

(23) Ganguly, N. C., Roy, S., Mondal, P., and Saha, R. (2012) An efficient one-pot organocatalytic synthesis of 9-(1H-indol-3yl)-xanthen-4-(9H)-ones under mild aqueous micellar conditions, Tetrahedron Lett. 53, 7067-7071.

(24) Trivedi, A. B., Kitabatake, N., and Doi, E. (1990) Toxicity of Dimethyl Sulfoxide as a Solvent in Bioassay System with HeLa Cells Evaluated Colorimetrically with 3-(4,5-Dimethyl thiazol-2-yl)-2,5-diphenyl-tetrazolium Bromide, Agric. Biol. Chem. 54, 2961-2966.

(25) Gülden, M., Jess, A., Kammann, J., Maser, E., and Seibert, H. (2010) Cytotoxic potency of $\mathrm{H}_{2} \mathrm{O}_{2}$ in cell cultures: Impact of cell concentration and exposure time, Free Radic. Biol. Med. 49, 1298-1305. 
(26) Singh, M., Sharma, H., and Singh, N. (2007) Hydrogen peroxide induces apoptosis in HeLa cells through mitochondrial pathway, Mitochondrion 7, 367-37. 